\title{
Población y desarrollo sostenible en México: revisión de sus relaciones complejas
}

\section{Population and sustainable development in Mexico: a review of its complex relationships}

\author{
Diego Abelardo González-Bejarano \\ Margarita Josefina Holguín-García \\ Facultad de Economía de la Universidad Autónoma \\ del Estado de México, México
}

Resumen

Este artículo tiene como objetivo revisar la relación entre la población y los indicadores del desarrollo para México en el periodo de 2010 a 2016. Iniciamos con la revisión del desarrollo conceptual, metodológico e instrumental aplicado a la experiencia del México reciente. Se propone una visión de conjunto de los conceptos e indicadores que constituyen un sistema que puede orientar la discusión respecto del avance en la consecución de objetivos y metas determinados de la población y el desarrollo sostenible de México. En términos simples el trabajo busca revisar qué variables se integran al binomio de la población y desarrollo sostenible. Para empezar, se sostiene que la dimensión demográfica está ausente en la discusión del desarrollo sostenible y que merece una revisión desde el punto de vista sistémico.

Palabras clave: Población, desarrollo sostenible, sistema e indicadores, relación y políticas.

Abstract

This article aims to review the population and development indicators for Mexico in the period from 2010 to 2016 . We begin with the review of the conceptual, methodological and instrumental development applied to the experience of recent Mexico. It proposes an overview of the concepts and indicators that constitute a system that can guide the discussion regarding the progress in the achievement of specific objectives and goals of the population and the sustainable development of Mexico. In simple terms, the work seeks to review which variables are integrated into the binomial of the population and sustainable development. To begin with, the demographic dimension is absent from the discussion of sustainable development and deserves a revision from a systemic point of view.

Keywords: Population, sustainable development, system and indicators, relationship and policies. 


\section{INTRODUCCIÓN}

$\mathbf{P}$

ero ¿cómo integrar a la población en el desarrollo sostenible? ¿Qué es?: integrar; ¿cómo considerar e incorporar múltiples interrelaciones entre la población y el desarrollo? ¿Cómo se hace? ¿Y cuáles son las modalidades de integración? Cómo lograr que la unidad de análisis fundamental sea el sistema sociodemográfico-sostenibilidad a escala nacional y sus subsistemas: tecnológico, económico, social, institucional y ambiental, y sus interrelaciones mutuas. Esto permite plantear la relación de estas dimensiones y sus indicadores para cada uno de los subsistemas de tal manera que no sea un análisis segmentado porque se ha dicho que la segmentación de las ciencias es un síntoma de su crisis para entender un sistema articulado en el contexto actual.

En ese sentido nuestra exposición se centra en principio en la discusión teórica de la población y el desarrollo sostenible, en seguida el marco de referencia internacional para revisar la relación entre población y desarrollo sostenible, en tercer lugar, hacemos un análisis de resultados de la relación población y desarrollo sostenible, por último, las conclusiones y de manera general se hacen propuestas de políticas públicas en términos generales.

\section{DiSCUSIÓN TEÓRICA DE LA POBLACIÓN Y DESARROLLO SOSTENIBLE}

El debate entre el crecimiento de la población y los recursos naturales ha sido histórico en el área de la demografía y la economía. Por ejemplo, se tiene esta preocupación desde tiempos de uno de los economistas clásicos, Robert Malthus (1798) hasta la Conferencia de Río para dar lugar a las nuevas preocupaciones desde el punto de vista sistémico. El estado actual del debate histórico, centra sus argumentos en el crecimiento cero: ¿una utopía deseable?, los límites del crecimiento y la capacidad de carga y la cuantificación del impacto de las actividades humanas: la "huella ecológica" (CEPAL, 2004)

Los tópicos de Robert Malthus, han tenido una continuidad hasta nuestros días, por lo que actualmente aún siguen con fuerza vigente. Sin embargo, hay que puntualizar que el debate sobre los recursos ha derivado, más que hacia una reflexión sobre su escasez o no, hacia el estudio de los impactos de la población en el medio ambiente y cómo estos pueden afectar la disponibilidad real de los recursos naturales (Mouds, 2018). En el contexto contemporáneo el pensamiento de Malthus ha sido utilizado para entender y estudiar el crecimiento de la población, así como para ar- 
gumentar que es la responsable o la causa de la mayor parte de los problemas medioambientales globales actuales del planeta: el agotamiento de los recursos, la malnutrición, el incremento de la contaminación, la deforestación y la desertificación.

Esta idea es expuesta gráficamente por Paul Ehrlich, autor del libro gráficamente titulado La "Bomba Demográfica" (1968). En él se plantea la fórmula I = PAT, según la cual el impacto ambiental (I) es el producto de la combinación del tamaño de la población $(\mathrm{P})$, el nivel de riqueza o consumo per cápita $(\mathrm{A})$, medida por el producto per cápita o por el nivel de consumo, y el nivel de tecnología o eficiencia de la producción (T). La fórmula, por tanto, relaciona los efectos independientes del tamaño de la población, el consumo y la tecnología como los principales determinantes del impacto ambiental (Ehrlich, 2010).

La mayor crítica que ha recibido esta perspectiva es la reducción de un fenómeno complejo como es el del impacto ambiental a una generalización cuantitativa, se omiten las características a escala local del uso de los recursos, criticando, a la vez, el hecho de que la fórmula relaciona factores que no son independientes, sino que están vinculados entre sí de manera compleja. Otra crítica es la formulada por el economista Julian Simon, citado en Marquette (1997), que defiende, siguiendo las ideas de Ester Boserup, la bondad del crecimiento, rebatiendo las tesis de los neomalthusianos argumentando que el precio de los recursos está bajando en la actualidad, de forma que el crecimiento de $(\mathrm{P})$ provoca un aumento en (T) que minimiza (I), como por ejemplo la búsqueda de nuevas fuentes energéticas, la mayor cantidad de los rendimientos agrícolas, entre otros y que hoy es debate de los gobiernos del mundo.

En cambio el esquema del marco sistémico e indicadores del "Proyecto evaluación de la sostenibilidad en América Latina y el Caribe", ESALC, 2007, plantea una alternativa de análisis y que es mucho más complejo pues indica que la sostenibilidad de América Latina debe incluir las siguientes dimensiones: el demográfico (sostenibilidad humana), el social (sostenibilidad social), la institucional (sostenibilidad institucional), el económico (sostenibilidad económica), el ambiental (sostenibilidad ecológica) y las relaciones externas (ambiente externo) (véase Esquema 1). Todo ello asociado a la capacidad de respuestas basadas en la adaptabilidad y capacidad (de respuesta) del individuo para hacer frente a los cambios sociológicos, económicos, tecnológicos, ambientales y sobre los factores externos y con ello reducir su vulnerabilidad. Es bajo esta perspectiva en que se ubica nuestro análisis de sus dimensiones e indicadores de la rela- 
ción población y desarrollo sostenible. Este enfoque es consistente y a fin con el Modelo Conceptual Presión-Estado-Impacto-Respuesta, Proyecto CIAT (Quiroga Martínez, 2007).

Esquema 1: Marco Sistémico e Indicadores ESALC

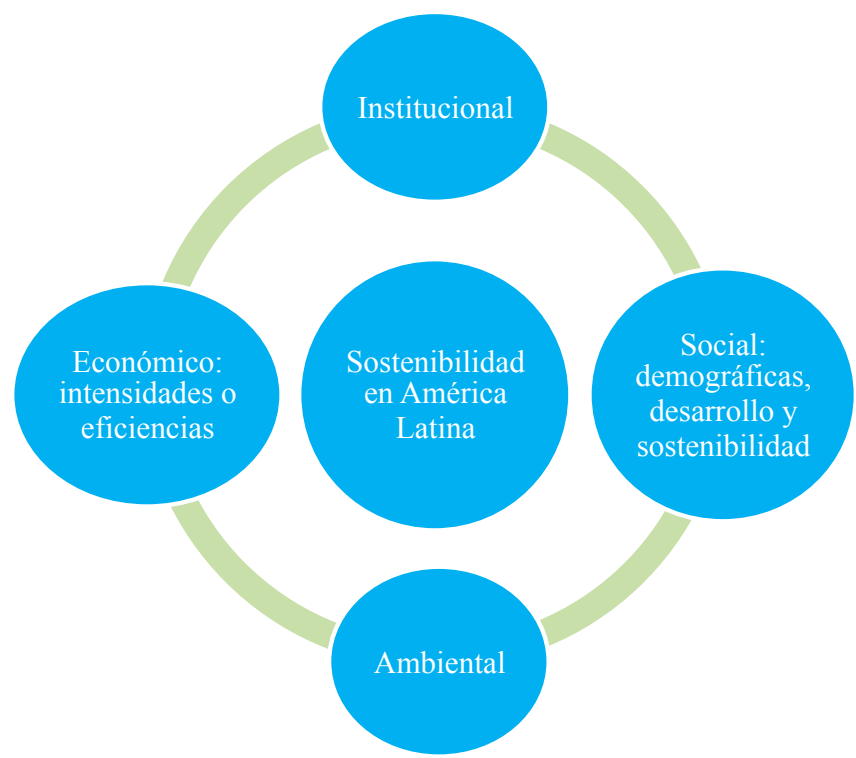

Fuente: elaborado con base en Rayén Quiroga Martínez, 2007, Indicadores ambientales y de desarrollo sostenible: avances y perspectivas para América Latina y el Caribe, Manual 55, disponible en https://repositorio.cepal.org/handle/11362/5498

Esta multidimensionalidad de la relación población y desarrollo sostenible permite ofrecer algunas pruebas empíricas, a la vez revisar sus alcances en términos de su utilidad para la política pública.

\section{Marco de REFERENCIA INTERNACIONAL PaRa Las POLÍTICAS DE POBLACIÓN Y DESARROLLO SOSTENIBLE}

Las propuestas de política entre población y desarrollo se remontan a las Reuniones de Roma, 1954 y Belgrado de 1965. Asimismo, tenemos la de Bucarest (1974): la Conferencia Mundial sobre Población; el Plan de Acción Mundial de Población. En la primera reunión fue de trascendencia mundial, porque el telón de fondo era el debate entre población y desarrollo en la cual se destacaba la estabilización demográfica en los países del 
norte como requisito para el desarrollo y los países del sur en desarrollo, la mejor receta era el uso de anticonceptivo para reducir el tamaño su población. Los principales aportes en la discusión de la interrelación de la población y desarrollo se destacaban la soberanía nacional en los temas de población, reconocimiento de derechos en asuntos demográficos, sobre todo el relacionado con la libre decisión del número y oportunidad en que se tienen los hijos, la promoción de la situación de la mujer, el establecimiento de metas genéricas, sobre todo en mortalidad y el relevamiento de políticas de población integradas a las de desarrollo (Welti, 2011).

En su caso la Conferencia Internacional sobre Población y las recomendaciones de la Ciudad de México (1984) se destacaba lo siguiente: se dio un giro en la decisión por el cambio en la postura de Estados Unidos, entonces se argumentaba que los problemas demográficos se solucionan con la acción del mercado; el crecimiento demográfico no tiene connotaciones negativas forzosas; los programas de planificación familiar no son la panacea; se rechaza de plano el aborto; las evidencias de cambio demográfico profundas aun presentaban ausencia en las políticas explicitas de población, con ello se recomendaba, la ampliación de las especificidades culturales que debían considerar la implementación de los programas internacionales en materia de población (Welti, 2011).

En la Conferencia Internacional sobre Población y el Desarrollo dio lugar al Programa de Acción de la Conferencia Internacional sobre Población y Desarrollo (CIPD, 1984) se destacaba lo siguiente: se puso énfasis en los derechos de los individuos; se concentró en ámbitos más íntimos como la sexualidad y las decisiones reproductivas; pero encontró resistencia al atarse a metas demográficas aunque todavía se usan propósitos estratégicos, como la estabilización de la población, y metas genéricas en morbimortalidad (infantil y materna), educación y accesos a la salud reproductiva (Naciones Unidas, 1994).

Asimismo, se privilegió la dimensión de género: además de empowerment of women se llama a la incorporación de los hombres en los programas de salud reproductiva y planificación familiar. La salud sexual y reproductiva como eje ordenador de los programas de población, a diferencia del enfoque antiguo de planificación familiar/salud materno/infantil. Pero, a la vez empezaron a privilegiar nuevos conceptos como la salud y derechos sexuales y reproductivos; el desarrollo sustentable; la inversión en recursos humanos; la equidad social, el advocancy (defensa); la especificación y relevamiento de grupos vulnerables de la población, como mujeres, adolescentes, indígenas, minusválidos. 
También se enfatizó en la consideración entre los problemas de población tradicionalmente excluidos como infanticidios, violación, el tráfico de mujeres, la explotación sexual, etc. en la importancia de las Organizaciones no Gubernamentales (ONG) y los actores no públicos en la concertación para el desarrollo a gran escala de programas de población (la noción del partnership); en la cuantificación financiera de los requerimientos de cooperación internacional, etcétera.

A finales del siglo XX Naciones Unidas, planteó objetivos denominados del milenio y se basaba en lo siguiente: objetivo 1: erradicar la extrema pobreza y el hambre; meta 2: reducir a la mitad entre 1990 y 2015 el porcentaje de personas con ingresos inferiores a un dólar; meta 3: reducir a la mitad, entre 1990 y 2015, el porcentaje de personas que padezcan hambre; objetivo 4: lograr la enseñanza primaria universal; meta 5: velar por que, para el año 2015, los niños y niñas de todo el mundo puedan terminar un ciclo completo de enseñanza primaria; objetivo 6: promover la igualdad entre géneros y la autonomía de la mujer; meta 7: eliminar las desigualdades entre los géneros en la enseñanza primaria y secundaria, preferiblemente para el año 2005, y en todos los niveles de la enseñanza antes del fin del año 2015; objetivo 8: reducir la mortalidad infantil; meta 9: reducir en dos terceras partes, entre 1990 y 2015, la mortalidad de los niños menores de cinco años.

Además se planteó objetivo 10: mejorar la salud materna; meta 11: reducir, entre 1990 y 2015, la mortalidad materna en tres cuartas partes; objetivo 12: combatir el VIH/SIDA, el paludismo y otras enfermedades; meta 13: haber detenido y comenzado a reducir, para el año 2015, la propagación del VIH/SIDA; meta14: haber comenzado a reducir, para el año 2015, la incidencia del paludismo y otras enfermedades graves; objetivo 15: garantizar la del medio ambiente; meta 16: incorporar los principios del desarrollo sostenible en las políticas y los programas nacionales e invertir la pérdida de recursos del medio ambiente; meta 17: reducir a la mitad, para el año 2015, el porcentaje de personas que carezcan de acceso sostenible a agua potable.

También se sugirió atender la meta 18: mejorar considerablemente, para el año 2020, la vida de por lo menos 100 millones de habitantes de tugurios; objetivo 19: fomentar una Asociación Mundial para el Desarrollo; Meta 20: en cooperación con los países en desarrollo, elaborar y aplicar estrategias que proporcionen a los jóvenes un trabajo digno y productivo; meta 21: en cooperación con las empresas farmacéuticas, proporcionar acceso a los medicamentos esenciales en los países en desarrollo; meta 22: en 
colaboración con el sector privado, velar por que se puedan aprovechar los beneficios de las nuevas tecnologías, en particular de las tecnologías de la información y de las comunicaciones. Estas metas marcaron las políticas de los países en desarrollo y que muchos de ellos han sido metas no cumplidas. Tal como sostiene Livi Bacci, 2014, esto es por no haber integrado los objetivos en un cuadro coherente, respetuoso del inseparable binomio desarrollo-sostentabilidad.

Hoy la sugerencia de los organismos internacionales se centran en los Objetivos de 2030, específicamente en las Metas e Indicadores del Desarrollo Sostenible (Agenda del Desarrollo post-2015 basado en lo siguiente: 1: fin a la pobreza; 2 : hambre cero; 3 : salud y bienestar; 4: educación de calidad; 5: igualdad de género; 6: agua limpia y saneamiento; 7: energía asequible y no contaminante; 8: trabajo decente y crecimiento económico; 9: industria, innovación e infraestructura; 10: reducción de las desigualdades; 11: ciudades y comunidades sostenibles; 12: producción y consumo responsable; 13: acción por el clima; 14: vida submarina; 15: vidas de ecosistemas terrestres; 16: paz, justicias e instituciones sólidas; 17: alianzas para lograr objetivos. Sobre estos objetivos es que hoy los gobiernos, incluyendo el de México, basan los planes nacionales y estatales, buscando responder a una política supranacional, a costa de sacrificar las necesidades de la población y el desarrollo interno en forma sostenible.

En palabras de Livi Bacci (2014), en general los documentos de los objetivos del Milenio y los de la Agenda después de 2015, no habían integrado aspectos económicos, sociales, ambientales ni los demográficos (argumento nuestro) y no se había afrontado nunca la necesidad de promover el desarrollo sostenible en la producción y el consumo al cual le damos la razón.

Para el caso de México, según Tudela (1993) y Carlos Welti (2011), la preocupación de la relación de la población y el desarrollo se inicia desde los tiempos Santa Ana hasta la actualidad, en cual el concepto de capacidad de carga es polémico (Welti, 2011). Pero Francisco Alba y Potter (1986) sostienen que el crecimiento demográfico y el desarrollo reciente se pueden ver desde el periodo de crecimiento económico sostenido: 1940-1970 cuya característica de poblar el país y un crecimiento económico sostenido (periodo reconocido como el de modelo sustitución de importaciones); posterior a los setentas (periodo de apertura comercial y crisis económicas recurrentes), se lleva a cabo la implantación del programa gubernamental y descenso de la fecundidad para lograr el objetivo de los 100 millones de habitantes (Ordorica, 1999) y posterior a esto a inicios del siglo XXI, en 
el cual se posiciona el denominado posmodernismo, cuya características es de la globalización y era de la información (Canales Cerón, 2003) y se destaca la ausencia de la planeación del desarrollo y el crecimiento demográfico (Welti, 2011).

\section{METODOLOGíA Y FUENTES DE INFORMACIÓN}

En este trabajo se hará uso del método científico, el cual, según Karl Popper, consiste básicamente en la formulación de ideas innovadoras y en su sometimiento a las más razonables, rigurosas y eficaces refutaciones posibles a través del método de ensayo y error: el método que consiste en proponer hipótesis y exponerlas a las más severas críticas, en orden a detectar dónde estamos equivocados (Molini Fernández s/f). Con base en ello, este artículo se somete a la crítica y que esperamos cumplir con las formulaciones, terminología, conceptos y contenido del desarrollo sostenible en el contexto de mayor cuestionamiento de la segmentación del análisis demográfico del mundo actual.

Para cumplir con los objetivos se valdrá de estadística básica los cuales buscarán describir y analizar las relaciones entre la población y el desarro1lo sostenible. Esquemáticamente las relaciones que tomarán en cuenta en este trabajo son la tecnología en el hogar, indicadores institucionales, los de sostenibilidad, el social, ambiental, externo el económico y el demográfico; lo que ESALC llama intensidades o eficacias.

Con base en estas dimensiones e indicadores es que se buscará probar las hipótesis de la multiplicidad de la relación causal de la población en el contexto del desarrollo sostenible. Para ello usaremos modelos de regresión múltiple porque permite obtener la relación causal combinada de la variable dependiente (Tasa de crecimiento demográfico TCP_P10-15) (Gujarati y Porter, 2009) y su relación con el sistema económico social.

La fuente de información que se usó para este trabajo permite el diseño del método mixto que se constituye, día a día, en una excelente alternativa para abordar temáticas de investigación en el campo de la Demografía y la Economía. Este texto plantea una revisión cuantitativa de las dimensiones como lo son la tecnología en el hogar, los indicadores institucionales, los de sostenibilidad, el social, el ambiental, el económico y el demográfico para verificar las intensidades o eficacias de la relación entre la población y el desarrollo sostenible del México reciente en las 32 entidades federativas. Es decir, nos basamos en datos de los censos de población, encuestas de la percepción (elemento subjetivo de los autores), fuentes de información económica, social y ambiental. 
Con esta información se corrió un modelo de regresión, el cual nos permite obtener relación entre la población e indicadores del desarrollo sostenible de las 32 entidades federativas de nuestro país (Tabla 1).

En términos de orden se expone lo siguiente: el crecimiento de la población esta correlacionada con las variables demográficas (crecimiento natural, crecimiento social, mortalidad infantil, esperanza de vida y envejecimiento), socioeconómicas (producto interno bruto per-cápita, participación económica femenina, relación de dependencia, pobreza) institucionales (confianza, corrupción), ambientales $\left(\mathrm{CO}_{2}\right.$, deforestación), externas (inversión extranjera directa y remesas) y tecnológicas (acceso a la tecnología en los hogares e indicadores de ciencia, tecnología e innovación). Todo ello implica una relación lineal y sistémica de la población y el desarrollo sostenible que se corrió con el paquete estadístico SPSS en su versión 21.

El modelo de regresión múltiple de la población y el desarrollo sostenible se expresa de la siguiente manera:

TCP_P10-15 (crecimiento demográfico) $=$ demográficas + socioeconómicas + institucional + ambiental + externas + tecnología del hogar (Tabla 1).

\section{ANÁLISIS SISTÉMICO DE RESULTADOS DE LA RELACIÓN POBLACIÓN Y DESARROLLO SOSTENIBLE}

\section{Bondad del ajuste}

En conjunto las dimensiones y variables explicatorias incluidas en el análisis de la población y el desarrollo sostenible explican un 84 por ciento en el modelo de la varianza de la variable dependiente (tasa de crecimiento de la población: TCP_P1015), pues R cuadrado corregida es igual a 0.836 . Además el error típico ha disminuido en 0.190 lo que indica una mejora en el ajuste. Cabe hacer notar que el valor corregido de $\mathrm{R}$ cuadrado no es igual al no corregido, pero sí para el $\mathrm{R}$ cuadrado y el cambio en $\mathrm{R}$ cuadrado (Tabla 2).

Gustavo Cabrera sostenía que la población debería ser un tema explícito de la política y para el caso de México, desde su independencia, se han desarrollado acciones legislativas concretas, y Leyes Generales de Población desde 1936, siempre con objetivos demográficos bien definidos. Pero ¿qué pasó? ¿en qué momento se desvinculó a la población, el desarrollo y la política? Se sostiene que desde que se instrumentó el modelo de política neoliberal al dejar todo a la libre fuerza del mercado (argumento neoclásico). 
Tabla 1: Marco sistémico e indicador de población con el desarrollo sostenible

\begin{tabular}{|c|c|}
\hline Variable dependiente (demográfica) & Variables explicatorias \\
\hline \multirow{25}{*}{$\begin{array}{l}\text { Tasa de crecimiento demográfico } \\
\text { TCP_P10-15 }\end{array}$} & a) Demográficas \\
\hline & Crecimiento natural $=\mathrm{CN}$ y social $=\mathrm{CS}(+)$ \\
\hline & Tasa de mortalidad infantilTMI2016 (-) \\
\hline & Índice de envejecimiento (\%envejecimiento 15$)(+)$ \\
\hline & Esperanza de vida (Esperanzae) (-) \\
\hline & b) Socioeconómicas \\
\hline & PIB per-capita (PIBpercapita15) $(+)$ \\
\hline & Relación de dependencia demográfica (TDEPEND15)(-) \\
\hline & \%de participación económica femenina (TasaOfem10) (-) \\
\hline & Porcentaje del comercio al por mayor (PCalpormayor15-16) (-) \\
\hline & Porcentaje del comercio al por menor (Pcmenor) $(+)$ \\
\hline & c) Institucional \\
\hline & Confianza en las instituciones \\
\hline & $\begin{array}{l}\text { ConfP1=mucha }(+) \text { Confp2=algo }(+), \text { Confp3=poca }(+) \text { y } \\
\text { Confp4=nada }(+)\end{array}$ \\
\hline & d) Ambiental \\
\hline & Emisión de dióxido de carbono $(\mathrm{CO} 2)(+)$ \\
\hline & Emisión de contaminantes antropogénicas (emiconA08) (-) \\
\hline & Emisión de contaminantes de fuentes naturales (emiconN08) $(+)$ \\
\hline & e) Externa \\
\hline & $\begin{array}{l}\text { Proporción de la inversión extranjera directa en el PIB (IEDPIB) (- } \\
\text { Remesas familiares de los migrantes internacionales (Remesas) } \\
(+)\end{array}$ \\
\hline & f) Tecnología en el hogar \\
\hline & $\%$ de usuarios internet (banda ancha) $($ Bancha15) $(+)$ \\
\hline & $\begin{array}{l}\text { \% de equipos de cómputo } \mathrm{x} \text { cada } 100 \text { hogares (EQcompu100H15) } \\
(-)\end{array}$ \\
\hline & \% de telefonía celular (Cel\%15) (-) \\
\hline & \% telefonía fija x cada 100 hogares (Telfija100H15) (-) \\
\hline
\end{tabular}

Nota: el signo negativo indica una relación negativa con la tasa de crecimiento de la población y el positivo es el caso inverso. Es este orden en que funcionan las hipótesis de este trabajo con perspectiva sistémica.

Fuente: elaboración propia con base en: http://www.cepal.org/deype/publicaciones/xml/4/34394/LCL2771e.pdf. 
Población y desarrollo sostenible en México: revisión de sus relaciones complejas / D.A. GONZÁLEZ Y M.J. HOLGUíN

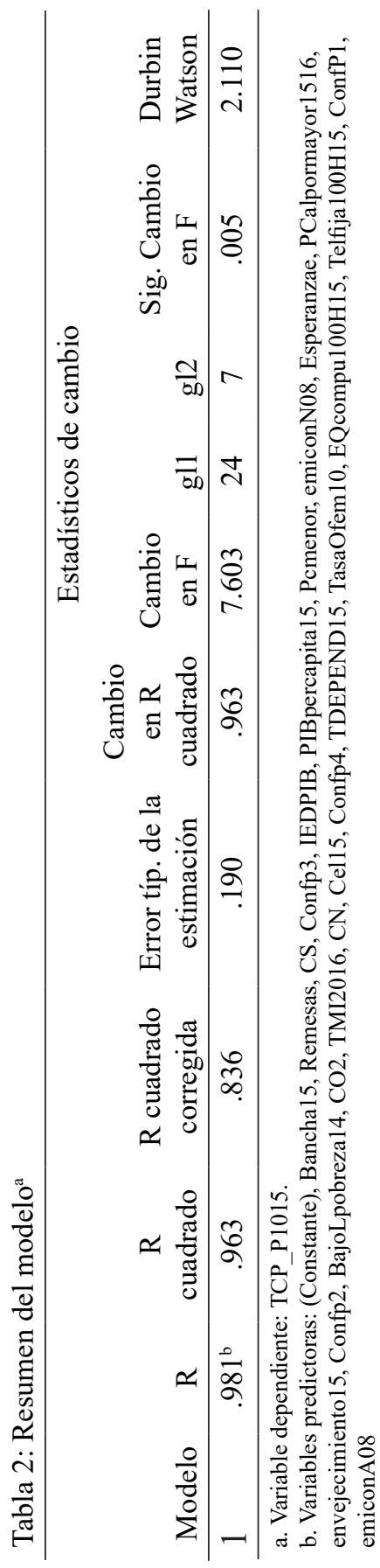


El logro de los objetivos de la política de población en nuestro país en su conjunto impactó positivamente en el desarrollo socioeconómico del país después de los setentas y las condiciones de vida de amplios sectores de la población; sin embargo, es difícil sostener que se ha avanzado significativamente en su consecución, más allá de disminuir el crecimiento de la población (en términos relativos) identificado en la ley como una acción para regularlo racionalmente y estabilizarlo pero se sabe poco de su relación con el desarrollo sostenible la cual es una relación multicausal (Welti, 2014).

En cuanto a los resultados derivados del modelo de regresión múltiple entre la población y el sistema complejo del desarrollo sostenible, se tienen lo siguiente:

La dimensión demográfica (crecimiento natural, crecimiento social, mortalidad infantil, esperanza de vida y envejecimiento), se puede argumentar lo siguiente: por cada unidad de cambio en la tasa de crecimiento natural (implica una disminución) la población disminuye en -0.00001899 (esto es gracias a la política de planificación familiar iniciada en 1974, pero no hay que olvidar que los adolescentes de este país son quienes hacen su mayor aporte en la fecundidad y por su puesto de la creciente mortalidad por causas degenerativas) (Ordorica, 2004); por cada unidad de cambio en la tasa de crecimiento social (aumento) la población crece en 0.0000007361 (la contribución anual del Saldo Neto Migratorios sucede de manera diferenciada al total de la población por entidad federativa, porque por un lado, tenemos saldos negativos derivados de la contra-urbanización, por otro, un crecimiento social alto) (Ibañez et al., 2015); por cada unidad de cambio en la tasa de mortalidad infantil (aumento), la población disminuye en -0.150 y sobre todo afecta la estructura de edad de la población según entidad federativa (CELADE, 1997); por cada unidad de disminución en la esperanza de vida, la población disminuye en -0.532 (las diferencias por entidad federativa está afectado por distintas causas y de las enfermedades crónicas degenerativas así como de los accidentes los cuales tienen implicaciones en su incremento o disminución) (Hernández y Narro; 2019); por cada unidad de aumento en el índice de envejecimiento la tasa de crecimiento de la población aumenta en 0.125 (Tabla 3). 
Población y desarrollo sostenible en México: revisión de sus relaciones complejas / D.A. GONZÁLEZ Y M.J. HOLGUíN

Tabla 3: Coeficientes ${ }^{\mathrm{a}}$

\begin{tabular}{|c|c|c|c|c|c|}
\hline \multirow[t]{2}{*}{ Modelo 1} & \multicolumn{2}{|c|}{$\begin{array}{l}\text { Coeficientes no } \\
\text { estandarizados }\end{array}$} & \multirow{2}{*}{$\begin{array}{r}\text { Coeficientes } \\
\text { tipificados } \\
\text { Beta }\end{array}$} & \multirow[b]{2}{*}{$\mathrm{t}$} & \multirow[b]{2}{*}{ Sig. } \\
\hline & $\mathrm{B}$ & Error típ. & & & \\
\hline (Constante) & -39.626 & 18.020 & & -2.199 & 0.064 \\
\hline $\mathrm{CN}$ & $-1.899 * 10^{-5}$ & 0.000 & -1.657 & -3.930 & 0.006 \\
\hline $\mathrm{CS}$ & $7.361 * 10^{-7}$ & 0.000 & 1.758 & 4.845 & 0.002 \\
\hline TMI2016 & -0.150 & 0.068 & -0.465 & -2.223 & 0.062 \\
\hline Esperanzae & -0.532 & 0.097 & -1.101 & -5.479 & 0.001 \\
\hline envejecimiento15 & 0.125 & 0.058 & 0.519 & 2.137 & 0.070 \\
\hline PIBpercapita15 & 0.002 & 0.001 & 0.377 & 1.796 & 0.116 \\
\hline PCalpormayor 1516 & -0.002 & 0.006 & -0.049 & -0.253 & 0.807 \\
\hline Pcmenor & 0.027 & 0.007 & 0.544 & 3.588 & 0.009 \\
\hline BajoLpobreza14 & 0.021 & 0.017 & 0.532 & 1.243 & 0.254 \\
\hline TDEPEND15 & -0.201 & 0.040 & -2.342 & -4.971 & 0.002 \\
\hline TasaOfem10 & -0.055 & 0.038 & -0.619 & -1.435 & 0.194 \\
\hline ConfP1 & 1.037 & 0.175 & 7.156 & 5.910 & 0.001 \\
\hline Confp2 & 0.980 & 0.199 & 2.994 & 4.937 & 0.002 \\
\hline Confp3 & 0.778 & 0.158 & 3.379 & 4.916 & 0.002 \\
\hline Confp4 & 1.254 & 0.207 & 6.800 & 6.051 & 0.001 \\
\hline $\mathrm{CO} 2$ & 0.003 & 0.001 & 11.192 & 3.275 & 0.014 \\
\hline emiconA08 & $-3.485^{*} 10^{-6}$ & 0.000 & -11.498 & -3.297 & 0.013 \\
\hline emiconN08 & $4.877^{*} 10^{-7}$ & 0.000 & 0.420 & 2.135 & 0.070 \\
\hline IEDPIB & -0.199 & 0.162 & -0.145 & -1.226 & 0.260 \\
\hline Remesas & 0.001 & 0.000 & 1.335 & 3.788 & 0.007 \\
\hline Cel15 & -0.030 & 0.020 & -0.564 & -1.497 & 0.178 \\
\hline Telfija100H15 & -0.038 & 0.015 & -1.252 & -2.527 & 0.039 \\
\hline EQcompu100H15 & -0.029 & 0.020 & -0.699 & -1.472 & 0.184 \\
\hline Bancha15 & 0.069 & 0.017 & 1.812 & 3.991 & 0.005 \\
\hline
\end{tabular}

a. Variable dependiente: TCP_P1015

Fuente: elaboración propia con fuentes mixtas. 
Los indicadores agregados derivados del modelo de regresión, en gran parte esconde las diferencias en el crecimiento demográfico de las entidades federativas que, se debe a la redistribución de personas y familias que buscan ampliar sus oportunidades de desarrollo económico y social; un ambiente que les permita mejorar su calidad de vida y escapar de las privaciones o falta de libertad, que impera en las regiones de origen (Sánchez et al., 2013); esto es porque el crecimiento demográfico de las entidades federativas ha estado marcado de manera importante por lo que hoy se llama contra-urbanización en los últimos 20 años, principalmente por la migración hacia el interior del país.

En la dimensión socioeconómica (producto interno bruto percápita, participación económica femenina, la relación de dependencia y la pobreza): resulta que el crecimiento demográfico no estimula el crecimiento económico, porque a cada unidad que aumenta el producto interno bruto percápita, la población tan solo lo hace en 0.002 , y esto prueba la hipótesis de que el crecimiento demográfico es un obstáculo al desarrollo económico de México (en todo caso el modelo de desarrollo excluye a la población y no es visto más que como unidad de consumo); a una unidad de cambio (disminución) al comercio al por mayor la población disminuye en - 0.002 ; para el cambio de una unidad al comercio al por menor, la población crece en 0.027 (las personas saben que las micro-empresas son generadoras de empleos y por ello el signo es positivo) (Morales, 2012); ante una unidad de cambio de la pobreza la población crece en 0.021 , esto tiene sentido porque en términos absolutos viven en zonas urbanas la mayor parte de los pobres de nuestro país (Coneval, 2016). A cada unidad de cambio en la tasa de dependencia económica la población decrece en - 0.201 (esto significa que hay proporcionalmente menos personas que constituyen una carga económica que debe ser solventada por la población en edad activa) (Chakiel, 2006); a cada unidad de cambio (aumento de la participación económica de la población femenina en el mercado laboral mexicano) la población decrece en -0.055 (Tabla 3). Pero dicha participación es cada vez más precaria y vulnerable al desempleo e informalidad de las mujeres (García Guzmán, 2013).

La dimensión institucional: la confianza, institucional de la población es importante para la población y el gobierno. El lastre más grande en los últimos tiempos ha sido la corrupción y su eficacia individual cuyo nivel es global, en este caso están más relacionadas con la percepción de las personas, y que a la vez creo que es una aventura relacionarla con el crecimiento de la población (Salama y Valier, 1995). Sin embrago, la falta de credibi- 
lidad de las instituciones son derivados de la gran desigualdad social (democracia cuestionada) prevaleciente en nuestro país y genera desconfianza institucional y sobre todo política (Cueto Villamán, 2007). En términos de las betas tenemos que cuando la gente confía mucho, cambia en una unidad, la población crece en 1.037; cuando cambia la categoría de confía algo la población crece 0.980 ; cuando la gente confía poco la población crece en 0.778; cuando uno confía nada la población crece en 1.254 (Tabla $3)$. Lo que significa es que las personas con independencia de la entidad de residencia aún confían en sus instituciones públicas en términos generales (pero prevalece la desconfianza para muchos) (Tronco, 2012). Obviamente esto hay un gradientes de desconfianza, principalmente hacia los partidos políticos. Con todo y eso aún confían en ellos, prueba de ellos es el porcentaje de personas que acudieron a votar para la reciente elección que es reconocido como anti-sistémico por el mismo presidente de la república Enrique Peña Nieto y dar lugar a lo que hoy llaman la cuarta transformación (cuyo modelo económicos no se sabe si es keynesiano u otro modelo).

La dimensión ambiental $\left(\mathrm{CO}_{2}\right.$, EmiconA08 y EmiconN08) desde la perspectiva ecológica sistémica sugiere trabajar en la reserva natural para tener un equilibrio ecológico-demográfico y económico. Ante cambios en una unidad de la misión de $\mathrm{CO}_{2}$, la población crece en 0.003 , esto tiene sentido porque según investigaciones recientes sostienen que emiten 100 empresas, 71 por ciento de gases de efecto invernadero en el país; esto es corroborado con el indicador de la emisión de contaminantes antropogénicas es negativa y baja, -0.000003485 ; así como la baja relación de la emisión de contaminantes de fuentes naturales que es de $0.0000004877 \mathrm{y}$ la población (Tabla 3). No quita, decir que también la población contamina a través del uso de automóvil, contamina el agua, el aire y en la producción de basura inorgánica (Hurtado Díaz, 2015). Que obviamente tiene sus efectos en la salud pública que también requieren de políticas de Estado, como de profesionales para prevenir y controlar enfermedades existentes o nuevas de la población.

La dimensión externa: inversión extranjera directa como proporción del PIB y las remesas, se tiene que cuando la inversión extranjera directa como proporción del PIB cambia en una unidad la población disminuye en -0.199 (es negativa); y el caso de las remesas enviadas por los migrantes internacionales desde el extranjero cambia en una unidad la población crece en 0.001 (Tabla 3), es decir, es muy poco el incentivo para que haga permanecer o crecer la población. 
La dimensión tecnológica: acceso a la tecnología en los hogares que es distinto a los indicadores de ciencia, tecnología e innovación. Ante el cambio en una unidad de uso del teléfono celular, la población decrece en -0.030 (esto tiene sentido porque según las encuestas disponible en México indican que 28.5 por ciento de la población no usa dicho medio de comunicación, con sus diferencias por entidad federativa); un cambio en una unidad de disponibilidad de teléfono fijo la población decrece en -0.038 (es entendible porque la población usa menos dicho medio de comunicación), por su parte ante un cambio en una unidad del equipo de cómputo la población disminuye en -0.029 (Tabla 3), pero se dice que aún en nuestro país existen 55.7 millones de personas usan computadora; en tanto que ante un cambio en la disponibilidad de banda ancha la población crece en 0.069 y para ello se dice que en nuestro país hay 62.4 millones utilizan Internet, pero cada vez, más el Internet móvil que no deja de ser desigual al interior del país.

\section{DiscuSIÓN Y CONCLUSIÓN}

En términos teóricos, la visión sistémica para analizar a la población y el desarrollo sostenible es amigable que puede evitar una visión fragmentada de los estudios de la población. Esto es porque la mayoría de los trabajos revisados sobre población se hace un corte y se verifican hipótesis fragmentados de la realidad poblacional, y para evitar esto la teoría de sistemas permiten articular la multidimensionalidad de la población y el desarrollo sostenible. En términos generales es un enfoque flexible (o lo que Gallopín dice que es un sistema abierto), al cual falta agregarle más dimensiones, variables e indicadores del desarrollo sostenible.

En términos metodológicos es un gran reto, porque en este ejercicio, hoy se usó el modelo de regresión múltiple, que también es flexible, pero podría ser otra técnica. Lo que sí es un gran desafío es la conciliación de los datos y la armonización de ellos.

Los resultados empíricos, son de gran valía al probar algunas hipótesis del modelo sistémico del crecimiento demográfico y sus consecuencias socioeconómicas, institucionales, ambientales, externas y el acceso tecnológico.

La dimensión del subsistema humano en sus determinantes, es afectado por el crecimiento natural que tiene una implicación inversa al crecimiento de la población, pero el social afecta en forma dinámica y positiva en la estructura de una población, el modelo de regresión apunta a ello. En este mismo subsistema, suele ser el factor dominante para la disminución de la 
tasa de crecimiento de la población por la mortalidad infantil, porque en el sistema demográfico expresa una salida. La esperanza de vida o expectativa moldea el crecimiento y cambio población de México en sus entidades federativas, por ejemplo, dichos cambios pueden verse reflejado en un mayor envejecimiento poblacional, lo cual se ve reflejado en la cúspide de la pirámide de población con diferencias en las entidades federativas.

La dimensión del subsistema socioeconómico, apunta a probar la hipótesis pesimista de escasa relación entre crecimiento demográfico y el económico de una relación no tan clara entre ambos subsistemas. Ahora resulta que los grandes comercio o el comercio al por mayor son antipoblacionistas (sobre todo sí se ubica en un contexto de crisis sanitaria como la de 2020) y eso tiene relación con los argumentos de un subsistema demográfico de la despoblación, es decir, el mercado incide de manera negativa en el crecimiento de la población, no así para el comercio al por menor, que sucede lo contrario. Como determinante del futuro de la población el comercio, en el caso de la urbanización, el análisis tenemos un distribución desigual (la distribución desigual de los factores de producción en el país) en la ventaja comparativa y la estructura del comercio. La tasa de dependencia demográfica significa que hay limitaciones por la baja participación laboral femenina, así como la extensión del periodo de formación y la participación laboral de adultos mayores. Y, esto nos lleva a un especial interés sobre el bono demográfico, su aumento relativo de la población en edades activas y las potencialmente activas con heterogeneidades por entidades federativas. Pero a la vez un rápido envejecimiento, contribuyera en un aumento de la población en general cuya relación es positiva en todo el país. Pero con mayor desigualdad y pobreza a tal grado que el Consejo Nacional de Evaluación de la Política de Desarrollo Social (CONEVAL) reconoce a 53.4 millones de personas en el país expresión de la gran desigualdad prevaleciente en México (CONEVAL, 2016).

El subsistema institucional es lo que se ha dado en llamar la postmodernidad sus indicadores muestra que ha generado desconfianza en las instituciones rectoras de la modernidad (más del Estado que del mercado). Se apuntan tres procesos que favorecen este hecho: la creciente individualización, el rechazo de la historia y la omnipresencia de la comunicación como proceso legitimador de las relaciones humanas que sesga la percepción de las personas hacia las instituciones (Martín Cabello, 2015).

El subsistema ambiental, la población urbana impone modelos de vida no acordes con las culturas de los pobres. Sin embargo, los más pobres denuncian que el deterioro ambiental se debe principalmente al consumo y 
despilfarro de recursos de los más ricos y que son ellos los que tienen que poner los medios para frenar el daño ambiental.

La dimensión de subsistema de población y relaciones externas: algunos autores coinciden en que la influencia de IED en la tasa de crecimiento del producto bruto real interno en términos per cápita se ejerce de dos formas: En primer lugar, a través del proceso de acumulación de capital que origina la entrada de este flujo de capitales en el país receptor. La IED impulsa el crecimiento incorporando nuevos inputs de tecnología más moderna en la función de producción de la economía receptora. En segundo lugar, influye en el crecimiento a través de la transferencia de conocimientos. Con frecuencia la IED incrementa el stock de conocimientos de la economía beneficiaria (sobre el capital humano y el empleo como proporción del total de la población aumenta), e introduce nuevas técnicas de organización empresarial, programas de aprendizaje, técnicas de marketing y otra serie de activos intangibles (sobre todo en lugares de escaso recurso para la investigación y desarrollo). También funciona como atractor de empresas nacionales y locales así como de población por ser generador de progreso social (Elías, Fernández y Ferrari, 2006).

La relación entre población y remesas monetarias de los migrantes internacionales significa que los bonos de la diáspora mexicana respaldados por las remesas, tienen el propósito de recaudar dinero con destino a proyectos de desarrollo al incentivar cadenas productivas locales (pero sobre todo para el gasto familiar), pero también para incentivar la retención y la reproducción de individuo en su lugar de origen.

El subsistema de la tecnológica en el hogar ocupa en la actualidad un importante espacio en la vida cotidiana de los mexicanos, pues son utilizadas en diversos contextos y con diferentes finalidades; no obstante, existe un sector de la población excluido de las oportunidades que las tecnologías de la información (TIC) expresados en la exclusión determinada por el nivel socioeconómico. Los resultados indican que el acceso a Internet y a las tecnologías está fuertemente segmentado; la estratificación digital va más allá del acceso y existen usos diferenciados con base en el capital cultural de la población según entidad federativa.

\section{Propuestas de política pública}

La propuesta es muy simple: primero que nada se tiene que tener un diagnóstico sistémico y con ello apoyar la planeación demográfica del país según sus diferencias estatales y regionales. Con ellos redactar el plan nacional de desarrollo (es decir privilegiar a la población en sus dimensión 
biológica-movilidad y social) y, en el cual, se especifique a la variable población como dependiente y sus relaciones complejas, es decir, privilegiar una articulación sistémica y no de manera segmentado obedeciendo a los criterios de instituciones internacionales como las del objetivo del milenio y los de 2030. Esto es porque el país tiene necesidades propias de su desarrollo demográfico, económico, social institucional y su articulación con el exterior. La decisión sistémica debe de partir de la necesidades de los más de 120 millones de mexicanos distribuidas en las 32 entidades federativas, de sus determinantes demográficos diferenciadas de sus efectos de la fecundidad y de su mortalidad. De los el efecto de la migración interna es la transformadora de la estructura poblacional, de sus jóvenes cuyo poder puede estructurar un nuevo poder en la ecuación de la producción y reconfigurar el país para prevenir un envejecimiento vulnerable. A la vez, que se tenga una fecundidad responsable acorde con los tiempos de la era de la información, así como cuidar la esperanza de vida y reducir la mortalidad infantil de la población más desprotegida del país.

Al tiempo de planificar el futuro del crecimiento de las ciudades y trabajar sobre los valores institucionales de la credibilidad a través de crear una cultura de la no corrupción e impunidad desde la familia, escuelas y las instituciones públicas. Es decir, debemos caminar hacia una nueva institucionalidad conectada a la población y no desconectada como siempre lo ha sido.

Trabajar para que el crecimiento de la población y el crecimiento económico logren una relación directa expresada en los beneficios del desarrollo que impacten en el bienestar de la población y eliminar las grandes brechas de la desigualdad social, lo cual se acentúa en una crisis prolongada.

Crear una cultura del cuidado del medio ambiente y evitar el despilfarro de recursos naturales. Pero, a la vez, crear una cultura del cuidado del aire, el agua, en general la tierra que es nuestra casa en la cual habitamos y encaminarse hacia la sostenibilidad.

Reducir la brecha al acceso tecnológico entre hogares y entre las entidades federativas. Pero sobre todo trabajar, en tecnología propia, más que hacer llegar tablets al resto de la población y para ello se requiere incrementar la inversión y financiamiento a la investigación y desarrollo en nuestro país. ¿Y por qué no pensar un nuestro valle de silicón mexicano? ¿O no se puede? Todo esto articulado a un modelo sistémico que corrija el rumbo demográfico y del desarrollo sostenible de nuestro país. 


\section{REFERENCIAS BIBLIOGRÁFICAS}

Alba, Francisco y Potter, Joseph, 1986, Población y desarrollo en México, una síntesis de la experiencia reciente, disponible en https://estudiosdemograficosyurbanos.colmex.mx/index.php/edu/article/view/568/561

Boserup, Ester, 1990, Economic and Demographic Relationships in Development (edited by T. Paul Schultz,). Baltimore: The Johns Hopkins University Press, disponible en https://www.cambridge.org/core/journals/ african-studies-review/ article/t-paul-schultz-ed-ester-boserup-economic-and-demographic-relationships-in-development-baltimore-and-london-the-johns-hopkins-university-press-1990-307-pp-bibliography-index-maps-tables-price-not-reported-paper/3D294931D50D89039E52B8AD99455BBE

Canales Cerón, Alejandro, 2003, Demografía de la desigualdad. El discurso de la población en la era de la globalización, disponible en https://www.researchgate. net/publication/281737633_Demografia_de_la_desigualdad_El_discurso_de_la poblacion_en_la_era_de_la_globalizacion

CELADE, 1997, 4. Mortalidad factores determinantes y consecuencias de las tendencias demográficas, vol. I (Naciones Unidas), consultado en https://repositorio.cepal.org/bitstream/handle/11362/34406/S9700582_es.pdf?sequence=1\&isAllowed $=\mathrm{y}$

CEPAL, 2004, Informe de la reunión de consulta sobre indicadores de desarrollo sostenible, Santiago de Chile, 7 al 9 de octubre del 2003, División de Desarrollo Sostenible y Asentamientos Humanos, LC/R.2120, marzo.

CEPAL, s/f, Acerca de Desarrollo Sostenible, disponible en https://www.cepal. org/es/temas/desarrollo-sostenible/acerca-desarrollo-sostenible.

CONEVAL, 2016, Medición de la pobreza, disponible en https://www.coneval. org.mx/Medicion/MP/Paginas/Pobreza-urbana-en-M\%C3\%A9xico-.aspx

Cueto Villamán, Francisco, 2007, “Desconfianza política, instituciones y gobernabilidad democrática en la República Dominicana”, en Ciencia y Sociedad, vol. 32, núm. 2, abril-junio, 2007, pp. 249-280. Instituto Tecnológico de Santo Domingo, Santo Domingo, República Dominicana, disponible en http://www.redalyc.org/ pdf/870/87032201.pdf

Chackiel, Juan, 2006, “América Latina: ¿hacia una población decreciente y envejecida?”, en Papeles de Población vol. 12 núm. 50, Toluca, octubre/diciembre.

Ehrlich, Paul, 2010, La bomba demográfica, disponible en https://apuntesdedemografia.com/2010/07/29/la-bomba-demografica-de-paul-ehrlich/

Elías, Silvina, Fernández, Rosario y Ferrari, Antonella, 2006, Inversión extranjera directa y crecimiento económico: un análisis empírico, disponible en http://www. aaep.org.ar/espa/anales/works06/Elias_Fernandez_Ferrari.pdf

Gallopín, Gilberto, 2003, Sotenibilidad y desarrollo sustentable: un enfoque sistémico, disponible en https://repositorio.cepal.org/bitstream/handle/11362/5763/1/ S033120_es.pdf 
García Guzmán, Brígida, 2013, Precariedad laboral y desempleo en México, disponible en https://archivos.juridicas.unam.mx/www/bjv/libros/8/3538/10.pdf

Gujarati, Domodar y Porter, Dawn, 2009, Econometría, disponible en https://scalleruizunp.files.wordpress.com/2015/04/econometria_-_damodar_n-_gujarati.pdf

Gustavo Cabrera Acevedo, 1990, El Estado mexicano y las políticas de población, disponible en https://ddd.uab.cat/pub/worpap/1990/184806/papersdemografia_a1990n47iSPA.pdf

Hernández-Bringas, Héctor H., 2019, José Narro-Robles, Mortalidad infantil en México: logros y desafíos, consultado en https://www.redalyc.org/jatsRepo/112/11262100002/html/index.html

Hurtado Díaz, Magali, 2015, La salud ambiental en México. Situación actual y perspectivas futuras, disponible en http://www.inegi.org.mx/eventos/2015/Poblacion/doc/p-MagaliHurtado.pdf

Livi-Bacci, Massimo, 2014, "Población y sustentabilidad: temas abiertos para el siglo XXI", en Papeles de Población, vol. 20, núm. 82, octubre-diciembre, pp. 1326, Universidad Autónoma del Estado de México, Toluca, México.

Marquette, Catherine, 1997, Turning but not Toppling Malthus: Boserupian Theory on Population and the Environment Relationships, disponible en http:// www. peakoilindia.org/wp-content/uploads/2013/10/Boserupian-Theory.pdf

Martín Cabello, Antonio, 2015, La desconfianza en las instituciones como expresión del cambio político y cultural, disponible en http://www.redalyc.org/ pdf/4959/495950263002.pdf

Molini Fernández, Fernando, s/f, Proactividad: el método cientifico de Karl Popper aplicado al futuro, disponible en http://www.encuentros-multidisciplinares. org/Revistan\%BA3/Fernando\%20Molini.pdf

Morales Díaz Covarrubia, René Rodrigo, 2012, Demografía de negocios en México, consultado en http://www.scielo.org.mx/scielo.php?script=sci_arttext\&pi$\mathrm{d}=$ S0186-72102012000100201\&lng=es\&nrm=iso

Mouds, 2018, Módulos Universitarios en Ciencia del Desarrollo Sostenible, disponible en http://www.desenvolupamentsostenible.org/es/poblacion-mundial-y-desarrollo-sostenible/4-la-interrelacion-entre-poblacion-y-medio-ambiente/4-2-el-estado-actual-del-debate-historico

Naciones Unidas, 1994, Informe de la Conferencia Internacional sobre la Población y el Desarrollo, El Cairo, 5 a 13 de septiembre, disponible en https:// documents-dds-ny.un.org/doc/UNDOC/GEN/N95/231/29/PDF/N9523129.pdf?OpenElement

Ordorica Mellado, Manuel, 1999, De la explosión a la implosión demográfica, disponible en estudiosdemograficosyurbanos.colmex.mx/index.php/edu/article/ download/.../1045

Ordorica Mellado, Manuel, 2004, “Cambios demográficos y desafíos para la política de la población en México. Una reflexión a largo plazo”, en Papeles de 
Población, vol. 10, núm. 40, abril-junio, p. 0 Universidad Autónoma del Estado de México Toluca, México

Partida Bush, Virgilio, s/f, Proyecciones de la población económicamente activa de México y de las entidades federativas, 2005-2050. Disponible en http://www. conapo.gob.mx/work/models/CONAPO/PEA/pea.pdf

Quiroga Martínez, Rayén, 2007, Indicadores ambientales y de desarrollo sostenible: avances y perspectivas para América Latina y el Caribe, Manual 55, disponible en https://repositorio.cepal.org/handle/11362/5498

Salama, Pierre y Valier Jaques, 1995, Corrupción y pobreza, consultado en http://148.202.18.157/sitios/publicacionesite/pperiod/espiral/espiralpdf/espiral4/45-68.pdf

Sánchez Rodríguez, Jesús Sergio, Luyando Cuevas, José Raúl, Aguayo Téllez, Ernesto, Picazzo Palencia, Esteban, 2013, "El desarrollo laboral sustentable y su relación con la migración interna en México", en Región y sociedad vol. 26 núm. 60 Hermosillo mayo/agosto 2014.

Simon, Julian, 1990, Population matters: people, resources, environment and immigration. New Brunswick, New Jersey: Transaction Publishers.

Tronco, José del, 2012, "Las causas de la desconfianza política en México", en Perf. latinoam. vol. 20 núm. 40, México, jul./dic., disponible en http://www.scielo. org.mx/scielo.php?script=sci_arttext\&pid=S0188-76532012000200009

Tudela, Fernando, 1993, Población y sustentabilidad del desarrollo: los desafios de la complejidad, disponible en http://revistas.bancomext.gob.mx/rce/magazines/250/1/RCE1.pdf

Ybáñez Zepeda, Elmyra, Gabriela Muñoz Meléndez, Rodolfo Cruz Piñeiro y Moisés Pérez Gamboa, 2015, "Algoritmo para estimar los Saldos Netos Migratorios en entidades federativas mexicanas", en Papeles de población vol. 21 núm. 83 Toluca ene./mar., consultado en http://www.scielo.org.mx/scielo.php?script=sci_arttext\&pid=S1405-74252015000100004

Welti Chanes, Carlos, 2014, "El Consejo Nacional de Población a 40 años de la institucionalización de una política explícita de población en México", en Papeles de Población, vol. 20, núm. 81, julio-septiembre, pp. 25-58 Universidad Autónoma del Estado de México, Toluca, México.

Welti-Chanes, Carlos, 2011, "La Demografía en México, las etapas iniciales de su evolución y sus aportaciones al desarrollo nacional", en Papeles de Población, vol. 17, núm. 69, julio-diciembre, 2011, pp. 9-47 Universidad Autónoma del Estado de México Toluca, México.

World Bank, 2018, Atlas 2018 de los Objetivos de Desarrollo Sostenible: una nueva guía visual sobre los datos del desarrollo, disponible en http://datatopics. worldbank.org/ sdgatlas/ 


\section{RESUMEN CURRICULAR DE LOS AUTORES}

Diego Abelardo González Bejarano

Egresado de la Facultad de Economía de la Universidad Autónoma del Estado de México. Es autor de fotografías orientadas a la migración internacional, las cuales han sido publicadas en libros dedicados a ello. Es autor de ensayos y artículos dedicados al envejecimiento y migración internacional, lo más reciente "Huellas de la migración centroamericana en el Estado de México" en la revista Sociedades y Desigualdades en 2019 y "Cambios en las causas de la emigración de los jóvenes mexicanos en el contexto de la crisis económica prolongada estadounidense y del México" en la revista COMMERCIUM PLUS en 2020.

Dirección electrónica: diego_neza@hotmail.com

Registro ORCID: https:/orcid.org/0000-0002-3197-4006

\section{Margarita Josefina Holguín García}

Es Profesora de Tiempo Completo de la Facultad de Economía de la Universidad Autónoma del Estado de México.

Dirección electrónica: margaritahg_96@yahoo.com.mx 